\title{
Review of Proximal Nail Antirotation (PFNA) and PFNA-2 - Our Local Experience
}

\author{
WL Loo, M Med Orth, SYJ Loh, FRCS (Edin), HC Lee, FRCS (Edin) \\ Department of Orthopaedic Surgery, Changi General Hospital, Singapore
}

\begin{abstract}
The proximal femoral nail antirotation (PFNA) device was recently introduced as an option for intramedullary management of pertrochanteric hip fractures. We report here local results with this relatively new implant. Sixty-two patients with pertrochanteric or subtrochanteric fractures underwent intramedullary fixation with PFNA devices in our centre during the 14 month period from September 2008 to October 2009. Data for this report were retrieved from clinical case records and operative notes. We recorded the age, gender, length of stay and operative time, preoperative ambulatory status and days required to regain mobility. Fractures were classified using the AO classification (A1, A2 and A3). Complications were also identified.There were 15 male and 47 female patients and the mean age was 74.3 years. The mean surgical duration was $76.1 \mathrm{~min}$ and the average length of hospitalisation was 14.5 days. Five cases were noted to have femoral shaft fracture and no occurrences of screw cutout. We found that $83.9 \%$ of the patients were able to regain preoperative mobility status by 6 months following surgery. We conclude that the PFNA is an effective implant for stabilisation of proximal hip fractures and that the local complication rate is similar to reports from other centres.
\end{abstract}

Key Words:

Hip Fracture, Intramedullary, Morbidity

\section{INTRODUCTION}

The treatment of intertrochanteric hip fractures and especially unstable intertrochanteric fractures in the elderly remains a challenge for orthopaedists. There is not a consensus of opinion as to the ideal implant for treatment of peritrochanteric fractures. The main goal of treatment is stable fixation that promotes early postoperative mobilisation. Extramedullary versus intramedullary devices for stabilisation of proximal hip fractures have been extensively reported in the literature ${ }^{1,2}$. Intramedullary devices appear to be highly appropriate due to their biomechanical properties. A Cochrane meta-analysis ${ }^{3}$ comparing extramedullary devices and older generation intramedullary nails concluded that the short term advantage of intramedullary fixation was negated by a higher incidence of operative morbidity.

Proximal femoral nail antirotation (PFNA) devices have been introduced as an intramedullary option in recent years (Figure 1). These devices were developed to obtain better fixation strength in the presence of osteoporotic bone and consist of an intramedullary nail with a proximal angulation of $6^{\circ}$ that is available in short and long versions. Compaction of cancellous bone by the helical blade into the femoral head increases rotational stability of cervicocephalic fragments and decreases load on the femoral head. Biomechanical testing has demonstrated that the blade has a significantly higher cut-out resistance than other commonly used screw systems. The PFNA blade may thus be a more biochemically suitable implant for unstable trochanteric fractures and trochanteric fractures associated with osteoporotic bone. Distal locking can be static or dynamic in such cases. The PFNA2 device was recently introduced and appears to be better suited to the typical Asian patient who has smaller femurs. We report here the results of our experience of PFNA and PFNA-2 in our centre.

\section{MATERIALS AND METHODS}

This retrospective study included all patients with intertrochanteric fractures, who were treated with PFNAs during the 14 month period from September 2008 to October 2009. Data were retrieved from case notes and operative records. Clinical and radiographic examinations were conducted on admission to the hospital, at 1 and 3 months post discharge and thereafter at 3-month intervals. A minimum follow-up of 6 months was required for inclusion in this study. Fractures were classified according to AO classification criteria. Operative time, early and late intraoperative and postoperative complications, and prefracture and postsurgical ambulatory status were analysed.

Surgery was carried out under general or spinal anaesthesia. All cases were either performed or supervised by an orthopaedic surgeon. A fracture table and image intensifier were used in all cases. All cases and x-rays were analysed by 
Table I: Analysis of PFNA and PFNA2 showed no difference in operative morbidity between the 2 implants.

\begin{tabular}{|lccc|}
\hline & Total & PFNA & PFNA2 \\
\hline Number & 62 & 21 & 41 \\
Gender & $15 \mathrm{M}: 47 \mathrm{~F}$ & $2 \mathrm{M}: 19 \mathrm{~F}$ & $13 \mathrm{M}: 28 \mathrm{~F}$ \\
Age & $76.4(22-99)$ & $80.6(59-99)$ & $74.3(22-93)$ \\
Mean days to op (days) & $3(1-10)$ & $3(1-9)$ & $4(1-10)$ \\
Mean length of stay (days) & $14.5(5-92)$ & $15(6-35)$ & $18.6(5-92)$ \\
Mean op time (min) & $76.1(25-225)$ & $70.4(25-120)$ & $80.4(35-225)$ \\
Anaesthesia & $16 \mathrm{GA}: 46 \mathrm{RA}$ & $4 \mathrm{GA}: 17 \mathrm{RA}$ & $12 \mathrm{GA}: 29 \mathrm{RA}$ \\
Femur fracture & 5 & 2 & 3 \\
Deep Wound infection requiring reoperation & 1 & 0 & 1 \\
\hline
\end{tabular}

a team of orthopaedic surgeons. Patients were referred to the physiotherapist and discharged to their own home or to a community hospital if further rehabilitation was required.

\section{RESULTS}

Sixty-two patients underwent stabilisation of pertrochanteric fractures during the 14 month study period. The mean patient age was 74.3 y (range: $22-99 y$ ), and the study sample comprised 15 male and 47 female patients. Twelve patients were diagnosed with ASA2, 46 were ASA 3 and 4 patients were ASA4 type fractures. There were 6(9.6\%) AO 31A1, forty-3 (69.3\%) AO 31A2 and 13 (20.9\%) AO31A3 fractures. The median number of days from admission to surgery was $3 \mathrm{~d}$ (range: 1-10d). The mean operative time was 76.1 minutes (range: 25-225min.). The median length of hospitalisation was 14.5d (range: 5-92d).

Sixteen patients had general anaesthesia and 46 opted for regional anaesthesia during surgery. The newer PFNA-2 was used in 41 patients, compared to 21 patients who had the original PFNA. This series consisted of 33 left and 29 right hips. The median helical blade length was size 10 (range: 912). The average drop in haemoglobin post-surgically was $1.99 \mathrm{~g} / \mathrm{dl}$.

Six patients were lost to follow up while 56 patients completed at least 6 months of follow up. Postoperatively, $60.7 \%$ of patients were ambulatory in the community and $35.7 \%$ were ambulatory but homebound. Two patients were wheelchair bound prior to their fracture. The median number of days to regain mobility was 9 days (range: $1-185 \mathrm{~d}$ ), and $83.9 \%$ of patients were able to regain mobility by 6 months postoperatively. Nine patients remained wheelchair bound at the six months follow up. Twenty (35.7\%) patients who were ambulatory in the community pre-fracture, with or without aid, regained their previous ambulatory status at six months. Eleven (19.6\%) patients regained independent ambulatory status, and $27(48.2 \%)$ patients were ambulatory at home with the aid of a walking frame(Figure $2 \mathrm{a}$ and $2 \mathrm{~b}$ ).

There were 4 cases of deep vein thrombosis, two of which led to pulmonary embolism. One patient passed away due to pneumonia. There were 3 cases of wound infection, one of which required multiple wound debridements and eventual removal of the helical blade. There were no cases of revision due to technical or implant failure and no reported case of helical blade cutout or penetration into the acetabular joint. However, there were 5 cases of femoral shaft fractures (Figure 3 )in this series, two of which required intraoperative conversion to a long PFNA and cerclage wiring. One patient with femoral shaft fracture showed non-union after six months of follow up. There were also three case of lateral protrusion of the sliding blade.

\section{DISCUSSION}

Trochanteric fractures occur mostly in elderly patients, and the outcome may be extremely poor if there is prolonged bedrest. Stable fixation that allows early mobilization is the treatment of choice. Opinions vary as to the best treatment for trochanteric fracture. Extramedullary devices such the dynamic hip screw are widely used locally although intramedullary nails are increasingly utilized for unstable trochanteric hip fractures due to their biomechanical advantages. Cemented hip hemiarthroplasties for intertrochanteric fractures have also been reported in the literature.

Intramedullary (IM) devices like the PFNA device and Gamma nails were not commonly used locally as they are associated with an unacceptable rate of cutout and femoral shaft fractures ${ }^{4,5}$, although other authors have reported excellent outcome with these IM nails ${ }^{6,7}$. The PFNA device was designed in light of these. The tip of the IM nail is specially designed to reduce the concentration of stress. When compared to a column screw, use of the helical blade results in increased contact surface area between the device and the femoral head cancellous bone, compressing rather than removing the limited amount of bone. The aim of this retrospective study was to evaluate this relatively new implant in our local population.

There was a predominance of female patients in our study, similar to others reports in the literature ${ }^{8,9}$. The mean patient age was $82 y$ in this study and the aetiology of most of the 


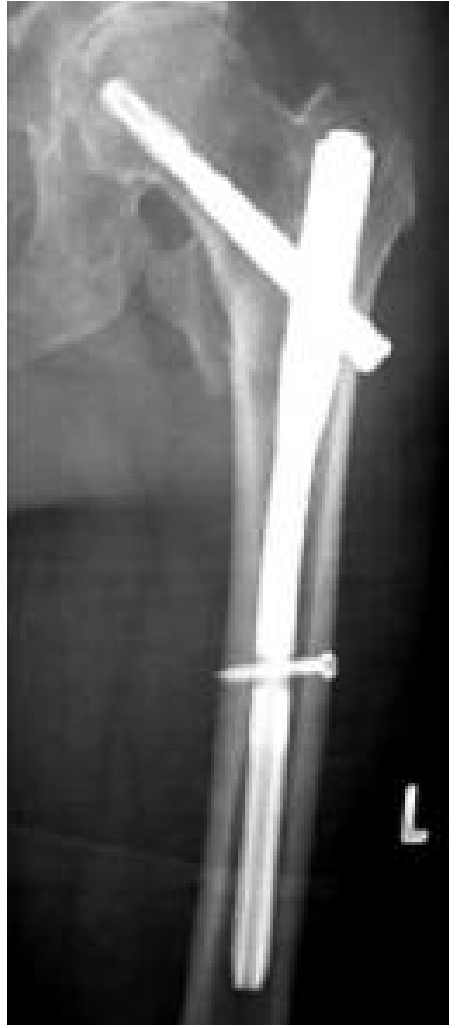

Fig. 1: The PFNA has a medial-lateral angle of $6^{\circ}$, which allows insertion at the tip of the greater trochanter. The flexible tip eases insertion and avoids stress on the bone at the tip of the implant.

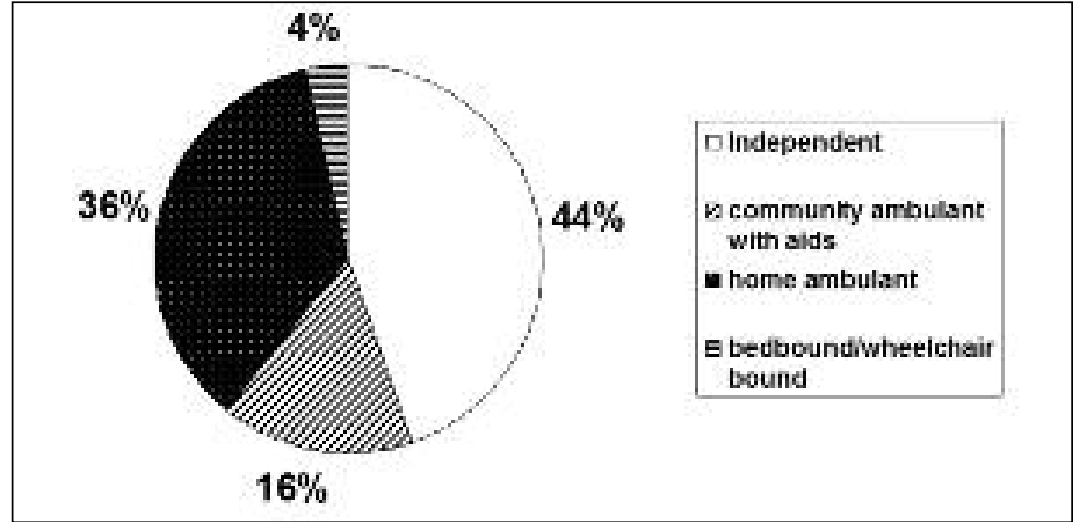

Fig. 2a: Represents pre-operative ambulatory status.

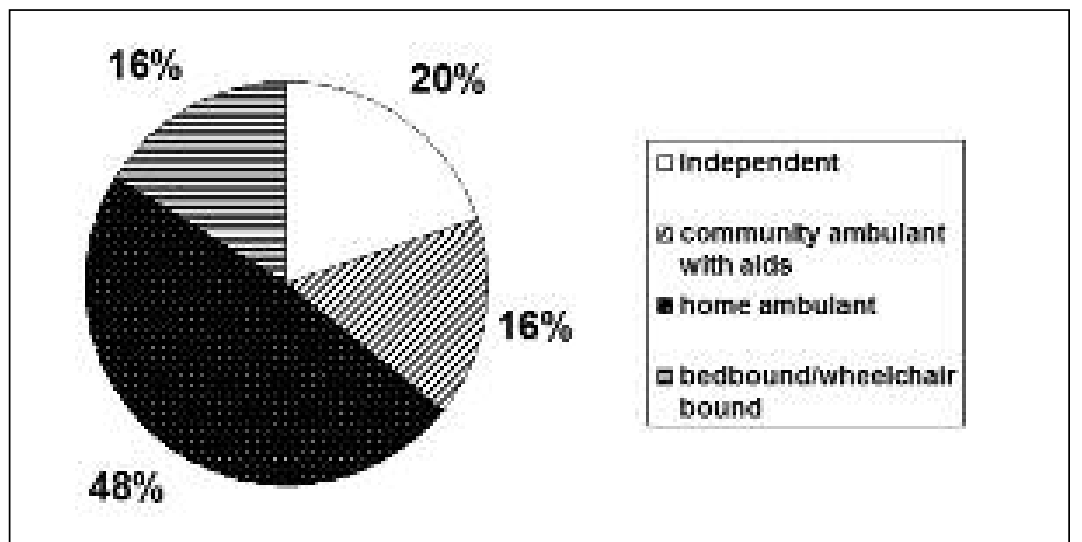

Fig. 2b: Represents ambulatory status at 6 months follow up. At 6 months, $84 \%$ of our patients were able to regain mobility.

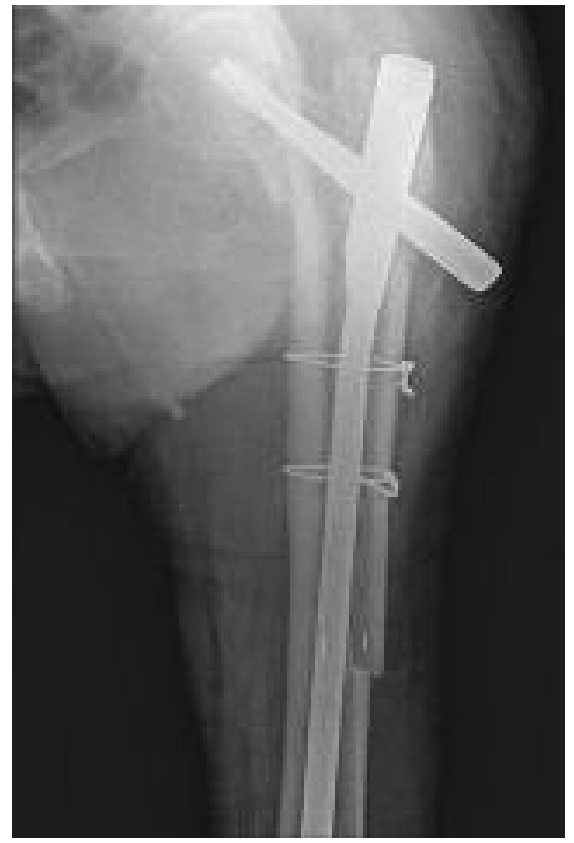

Fig. 3: X-Ray showed fracture of the femoral shaft fracture during PFNA stabilization of intertrochanteric fracture and lateral protusion of the nail. fractures was low-energy in nature, consisting of a domestic fall. There were no instances of helical blade penetration into the acetabulum or cut out in this study, a result similar to those reported in the literature ${ }^{10}$. There was three case of lateral protrusion of the sliding blade in the present series. Of note, there were 5 cases of femoral shaft fractures and two cases requiring intraoperative conversion to a long PFNA and cerclage wiring. Technical considerations included the canal diameter of the proximal femur, excessive femoral antecurvatum and unrecognized fracture propagation down the shaft during nail insertion.

There were 3 cases of wound infection among the patients analysed, one deep and two superficial infections. The deep infection case required multiple debridements and removal of the helical blade. The limited exposure and decrease in needed muscle release when utilising a PFNA device compared to extramedullary devices like the dynamic hip screw (DHS) can partly explain the relatively low rate of infection. Our complication rates were similar to those reported in the literature ${ }^{11,12}$. 
One limitation of the current study is its small sample size. Moreover, this study involves several surgeons who needed to overcome the learning curve of this relatively new implant. The implant is also more costly compared to the DHS. Randomised controlled trials are needed to more extensively and accurately compare the PFNA device to the DHS screw. Appropriate patient selection and recognition of femurs with narrow canal or too much bowing are required to ensure optimal results. However, it is encouraging to see that $83.9 \%$ of our PFNA patients were able to regain preoperative ambulatory status at 6 months following surgery. Some patients required some form of walking aid due to fear of walking and/or weakened musculature.

Good reduction of the fracture, and optimal positioning and length of the hip helical blade are crucial to achievement of good outcomes with the PFNA device. Further studies are needed to compare this new implant with extramedullary devices ${ }^{14}$. 


\section{REFERENCES}

1. Butt MS, Krikler SJ, Nafie S, Ali MS. Comparison of dynamic hip screw and gamma nail: a prospective, randomized, controlled trial. Injury 1995; 26: 615-8.

2. Schipper IB, Marti RK, van der Werken C. Unstable trochanteric femoral fractures: extramedullary or intramedullary fixation. Review of literature. Injury 2004; 35(2): 142-51.

3. Parker MJ, Handoll HH. Gamma and other cephalocondylic intramedullary nails versus extramedullary implants for extracapsular hip fractures in adults. Cochrane Database Syst Rev 2008; (3): CD000093.

4. Albareda J, Laderiga A, Palanca D, Paniagua L, Seral F. Complications and technical problems with gamma nail. Int Orthop 1996; 20: 4750.

5. Herrera A, Domingo LJ, Calvo A, Martinez A. A comparative study of trochanteric fractures treated with Gamma nail or the proximal femoral nail. Int Orthop 2002; 26: 365-9.

6. Domingo LJ, Cecilia D, Herrera A, Resines C. Trochanteric fractures treated with a proximal femoral nail. Int Orthop 2001; 25: 298-301.

7. Al-yassari G, Langstaff RJ, Jones JW, Al Lami M. The AO/ ASIF proximal femoral nail (PFN) for the treatment of un stable trochanteric fracture. Injury 2002; 16: 386-93.

8. Simmermacher RKJ, Bosch AM, Van der Werken C. The AO/ASIF proximal femoral nail (PFN): a new device for the treatment of unstable proximal femoral fractures. Injury 1999; 30: 327-32.

9. Sermon A, Broos PLO. The use of the proximal femoral nail antirotation in the treatment of intertrochanteric hip fractures: clinical and radiological results in 63 patients. Folia Traumatologica Lovaniensia 2007; 48-56.

10. Brunner A, Jöckel JA, Babst R. The PFNA proximal femur nail in treatment of unstable proximal femur fractures-3 cases of postoperative perforation of the helical blade into the hip joint. J Orthop Trauma 2008; 22(10): 731-6.

11. Mereddy P, Kamath S, Ramakrishnan M, Malik H, Donnachie N. The AO/ASIF proximal femoral nail antirotation (PFNA): a new design for the treatment of unstable proximal femoral fractures. Injury 2009; 40(4): 428-32.

12. Simmermacher RK, Ljungqvist J, Bail H, Hockertz T, Vochteloo AJ, Ochs U, et al. The new proximal femoral nail antirotation (PFNA) in daily practice: results of a multicentre clinical study. Injury 2008; 39(8): 932-9.

13. Takigami I, Matsumoto K, Ohara A, Yamanaka K, Naganawa T, Ohashi M, et al. Treatment of trochanteric fractures with the PFNA (proximal femoral nail antirotation) nail system - report of early results. Bull NYU Hosp Jt Dis 2008; 66(4): $276-9$.

14. Zou J, Xu Y, Yang H. A Comparison of Proximal Femoral Nail Antirotation and Dynamic Hip Screw Devices in Trochanteric Fractures. J Int Med Res 2009; 37(4): 1057-64. 